\title{
Classical dendritic cells mediate fibrosis directly via the retinoic acid pathway in severe eye allergy
}

\author{
Sarah D. Ahadome, ${ }^{1,2}$ Rose Mathew, ${ }^{1}$ Nancy J. Reyes, ${ }^{1}$ Priyatham S. Mettu, ${ }^{1}$ Scott W. Cousins, ${ }^{1,3}$ \\ Virginia L. Calder, ${ }^{2}$ and Daniel R. Saban ${ }^{1,3}$ \\ 'Department of Ophthalmology, Duke University School of Medicine, Durham, North Carolina, USA. ${ }^{2 D e p a r t m e n t ~ o f ~}$ \\ Ocular Biology and Therapeutics, University College London (UCL) Institute of Ophthalmology, London, United Kingdom. \\ ${ }^{3}$ Department of Immunology, Duke University School of Medicine, Durham, North Carolina, USA.
}

\begin{abstract}
Fibrosis is a shared end-stage pathway to lung, liver, and heart failure. In the ocular mucosa (conjunctiva), fibrosis leads to blindness in trachoma, pemphigoid, and allergy. The indirect fibrogenic role of DCs via T cell activation and inflammatory cell recruitment is well documented. However, here we demonstrate that DCs can directly induce fibrosis. In the mouse model of allergic eye disease (AED), classical CD11 b+ DCs in the ocular mucosa showed increased activity of aldehyde dehydrogenase (ALDH), the enzyme required for retinoic acid synthesis. In vitro, CD11b+ DC-derived ALDH was associated with 9-cis-retinoic acid ligation to retinoid $x$ receptor (RXR), which induced conjunctival fibroblast activation. In vivo, stimulating RXR led to rapid onset of ocular mucosal fibrosis, whereas inhibiting ALDH activity in DCs or selectively depleting DCs markedly reduced fibrosis. Collectively, these data reveal a profibrotic ALDH-dependent pathway by DCs and uncover a role for DC retinoid metabolism.
\end{abstract}

Conflict of interest: SDA is an inventor of a Patent PCT/CB2015/051292 held by UCL Business PLC.

Submitted: February 9, 2016 Accepted: May 26, 2016 Published: August 4, 2016

Reference information: JCI Insight. 2016;1(12):e87012. doi:10.1172/jici.insight.87012

\section{Introduction}

Fibrosis, or excessive collagen deposition that leads to permanent scar formation, is a common pathologic pathway in organ failure such as in renal fibrosis, cirrhosis, and chronic obstructive pulmonary disorder. Likewise, fibrosis of the ocular mucosa (i.e., conjunctiva) is a clinically significant problem, which leads to blindness in advanced forms of eye diseases, including trachoma, mucous membrane pemphigoid (MMP), and Stevens-Johnson syndrome. Fibrosis is also common in chronic allergic diseases (1-3), which include allergic bronchial asthma, atopic dermatitis, and atopic keratoconjunctivitis (4-6). The fibrogenic nature of allergic inflammation, including in allergic eye disease (AED), has been attributed to the Th2 lymphocyte cytokine IL-13 (1, 2, 5).

In addition, retinoic acid (RA) has received considerable attention in fibrosis pathobiology (7-10). Retinoid/vitamin A metabolites such as RA are important biological molecules involved in vision, development, cell growth and maturation, apoptosis, and immunologic function. RA is also recognized for its role in fibroblast growth and proliferation and production of extracellular matrix proteins such as collagen (11-14). However, under certain pathologic settings, RA can exert profibrotic effects, as evidenced by hepatic stellar cells and liver fibrosis, as well as renal fibroblasts $(7,9,10)$.

The emerging role in RA synthesis for DCs has been a topic of intense investigation recently, with attention mostly focused on RA in immunologic homeostasis and lymphocyte homing within the small intestine lamina propria and gut-associated lymphoid tissues (15-21). As a result, classical DCs (cDC) are now recognized as important producers of the aldehyde dehydrogenase 1 (ALDH1) family. These enzymes are required in the oxidation step to convert retinal into all-trans-RA (ATRA) or isomerized 9-cis-RA (9-CisRA), which consequently signal to target cells in a paracrine manner through nuclear RA receptor (RAR) and retinoid $x$ receptor $(\mathrm{RXR})(22,23)$. Recent reports also confirm the relevance of RA synthesis by cDCs in other organs, such as the skin $(24,25)$. Furthermore, transcriptome data available through the Immunological Genome Project Consortium (https://www.immgen.org/) corroborates that certain cDCs in liver and lung also express high levels of Aldh1a2 mRNA (26), which may suggest that RA synthesis by nonlymphoid tissue $\mathrm{cDCs}$ is a generalized phenomenon. 
Given the current understanding for $\mathrm{cDC}$-derived ALDH in RA synthesis, in conjunction with the appreciable role of RA on fibroblast function, we herein examined whether $\mathrm{CDCs}$ have a direct fibrogenic potential in the ocular mucosa by virtue of ALDH produced by these cells. We took advantage of the established mouse model of AED, which replicates sight-threatening allergies such as in atopic keratoconjunctivitis and is often associated with conjunctival fibrosis (27). Our results reveal a particular subset of cDCs (i.e., $\mathrm{CD} 11 \mathrm{~b}^{+}$or $\mathrm{CDC} 2$ ) (28) with increased ALDH activity that causes ocular mucosal fibrosis in AED, and they implicate that this pathway is mediated via fibroblast RXR ligation of RA. Importantly, we were able to demonstrate that selective depletion of mouse DCs in vivo, or inhibition of $\mathrm{cDC}$-derived ALDH in vivo, led to the protection from fibrosis during AED. Our results are supported by the companion article by Dart and colleagues (29), who reveal a profibrotic autocrine mechanism mediated by fibroblast-derived ALDH in progressive scarring of conjunctiva in patients with MMP - a prototypic human autoimmune mucosal scarring disorder.

In summary, results here demonstrate that $\mathrm{cDC}$-derived ALDH contributes to ocular mucosal fibrosis in allergic inflammation. Our findings are novel to our knowledge in that DCs are shown herein to induce fibrosis in a direct manner. Direct, cDC2-induced fibrosis is distinct from indirect fibrogenesis, which is mediated by activation or recruitment of immune cells in chronic inflammation that in turn causes fibrosis. We showed that DCs directly mediate fibrosis via RA synthesis. Indeed, both our study and the companion article converge on the finding that ALDH/RA plays a pivotal role in fibrosis of the ocular mucosa. Thus, our work has the potential to open up a new area for fibrosis research in the cDC-mediated metabolism of retinoids and the possibility for therapeutic strategies in combating fibrotic disease in eye and perhaps elsewhere. Future work is required to determine whether $\mathrm{cDCs}$ in humans likewise mediate fibrosis of the ocular mucosa in allergy and whether our findings also extend to other fibrosis in other etiologies, such as trachoma.

\section{Results}

Uniquely high ALDH activity in steady-state $c D C 2$ s is conserved in multiple tissues. The ocular mucosa harbors steady-state $\mathrm{cDC} 1$ (i.e., $\mathrm{CD}_{103^{+}}$) and $\mathrm{CDC} 2$ (i.e., $\mathrm{CD}_{11 b^{+}}$) (30), and our first aim was to measure these cells at the level of the conjunctiva and draining submandibular/cervical lymph nodes (LNs) (Figure 1, A and B) for their ALDH activity. We also compared steady-state CDC1 and CDC2 from the lung as a control (Figure 1C). Positive ALDH activity in these cells was determined using ALDEFLUOR staining, whereas negative controls for ALDH activity were accomplished by ALDEFLUOR staining in the presence of the ALDH inhibitor diethylaminobenzaldehyde (DEAB). Data show that a large fraction of $\mathrm{CDC} 2$ at all sites were $\mathrm{ALDH}^{+}$, whereas only a small fraction of $\mathrm{cDC} 1$ were positive - and only at some sites (Figure 1, D and E). Furthermore, we were able to demonstrate that the transcriptome data available by Heng et al. from the Immunological Gene Network Consortium (26) show a similar trend for Aldh1a2 mRNA levels in CD11b cDCs from the lung, liver, and skin (Supplemental Figure 1; supplemental material available online with this article; doi:10.1172/jci.insight.87012DS1). Together, our findings led us to conclude that ALDH activity is uniquely high in ocular mucosal $\mathrm{CDC} 2$ and that this profile is conserved in multiple tissues.

$A L D H$ activity from $C D 11 b^{+} B M$-derived $D C s$ is fibrogenic via $R A$ activation of $R X R$ in conjunctival fibroblasts. Based on our finding that $\mathrm{CDC} 2 \mathrm{~s}$ harbor uniquely high ALDH activity, we next wanted to investigate the potential effect of cDC2-derived ALDH activity on fibrogenesis. To address this question, we took advantage of $\mathrm{CD}_{11 \mathrm{~b}^{+}}$and $\mathrm{CD} 103^{+} \mathrm{BM}$-derived DCs (BMDCs), which we generated with high yields using granulocyte-macrophage CSF (GM-CSF) and FMS-like tyrosine kinase 3 (Flt-3), respectively $(25,30,31$ ). Similar to Helft et al. (32), GM-CSF generated BMDCs resulted in a large fraction of I-A/I-E bright cells and a smaller fraction of I-A/I-E intermediate cells (Supplemental Figure 2). We verified that, like cDC2s, $\mathrm{CD}_{11 b^{+}}$BMDCs harbored high ALDH activity, while CD103+ BMDCs did not (Figure 2, A and B), as reported by Yokota et al. (25).

Next, we transferred conditioned media from these respective BMDCs into cultures of primary conjunctival fibroblasts and subsequently assayed contractile and proliferative capacities of these fibroblasts. Data show that, compared with untreated fibroblasts, those receiving CD $11 b^{+}$BMDC-conditioned media had significantly lower levels of contraction and proliferation (Figure 2C). We considered reduced proliferation and contraction of conjunctival fibroblasts to be indicative of a profibrotic state in the conjunctiva as a result of the findings in the companion study. In their study, the profibrotic phenotype, demonstrated by explanted ocular MMP fibroblasts in vitro, showed a significant increase in both 
A Oc Mucosa

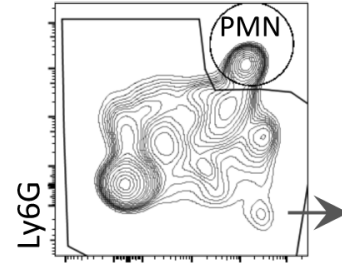

Ly6C

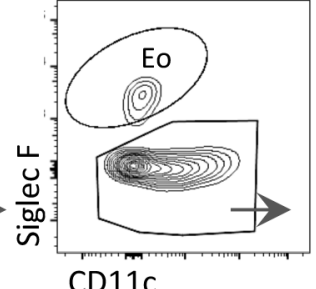

CD11c

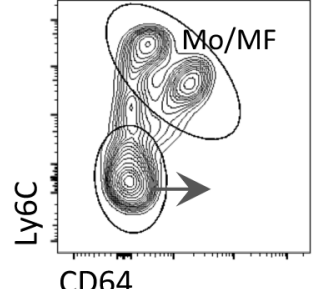

CD64

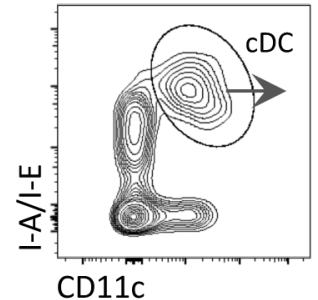

CD11c

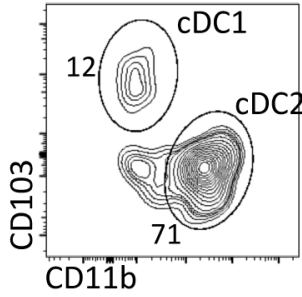

CD11b

\section{B LN (Cervical/Submandibular)}

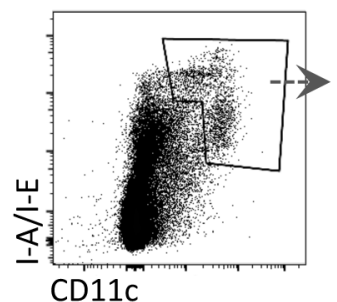

C Lung

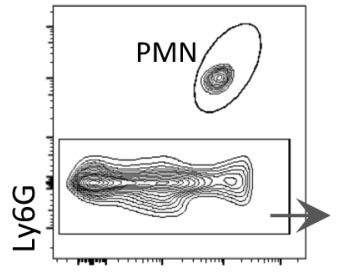

Ly6C
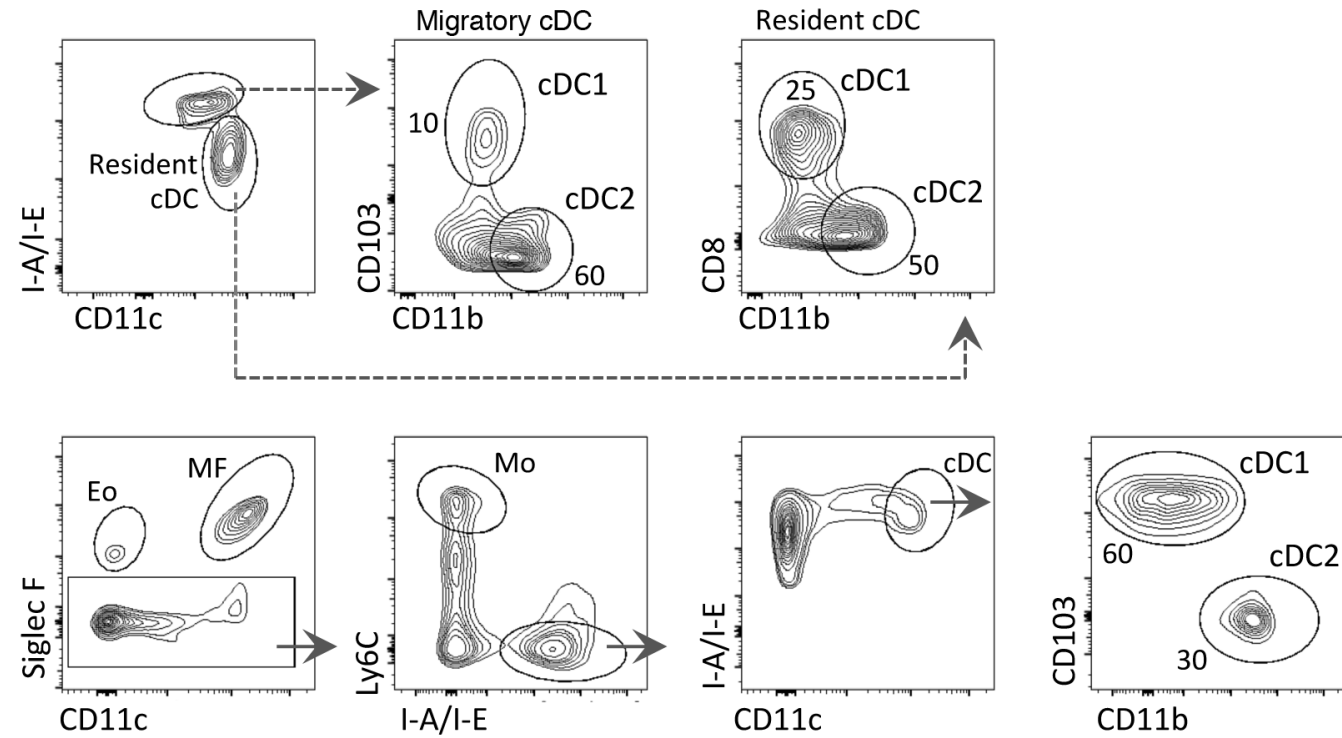

CD11b

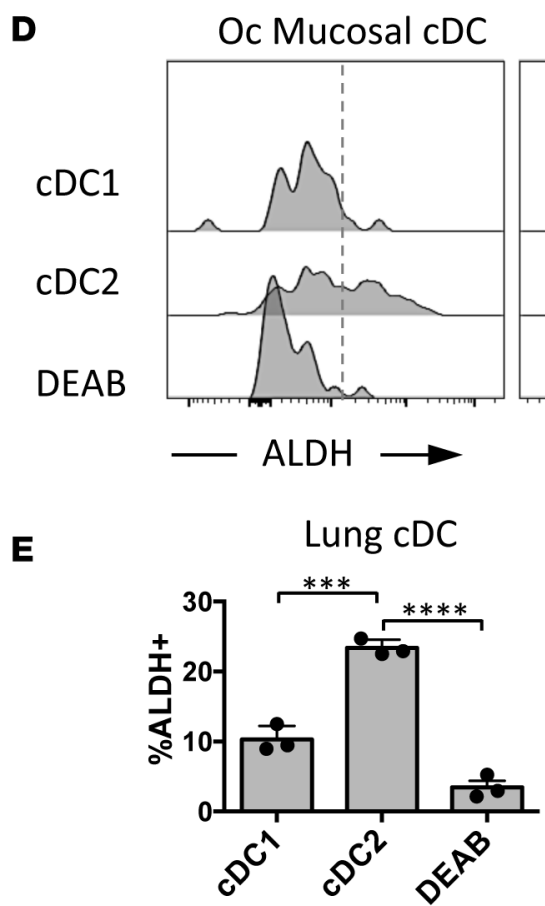

\section{LN Migratory CDC}

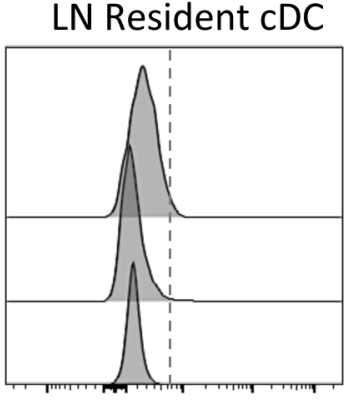

Lung CDC
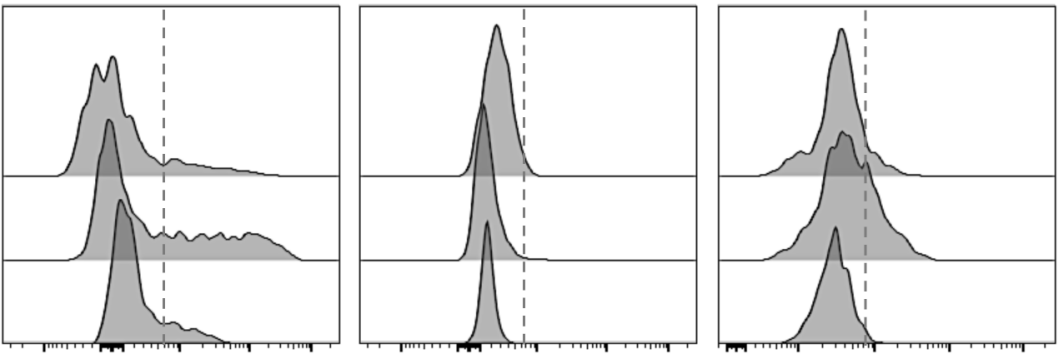

Figure 1. Uniquely high aldehyde dehydrogenase (ALDH) activity in steady-state type-2 classical DCs (CDC2) is conserved in multiple tissues. (A-C) Gating strategies for analyzing steadystate $\mathrm{CDC1}$ and $\mathrm{CDC2}$ in ocular (Oc) mucosa, draining submandibular/cervical lymph node (LN), and lung from normal C57BL/6 mice (PMN, neutrophils; Eo, eosinophils; Mo/MF, classical monocytes and macrophages). (D and E) Comparison of ALDH activity in CDC1s and cDC2s. Cell suspensions were stained with ALDEFLUOR to assess ALDH activity. Negative controls are shown for the respective $C D C 2$ populations, which were ALDEFLUOR-stained samples treated with the ALDH inhibitor diethylaminobenzaldehyde (DEAB). Data are representative of duplicate data points from 2 separate experiments on pooled conjunctiva from $n=10$ mice. Lung data is representative of triplicate data points from 3 separate samples from $n=20$ mice (error bars represent \pm SEM, ${ }^{* * *} P<0.0005,{ }^{* * *} P<0.00005,1$-way ANOVA with Bonferroni correction). Each experiment was carried out at least twice. 
A

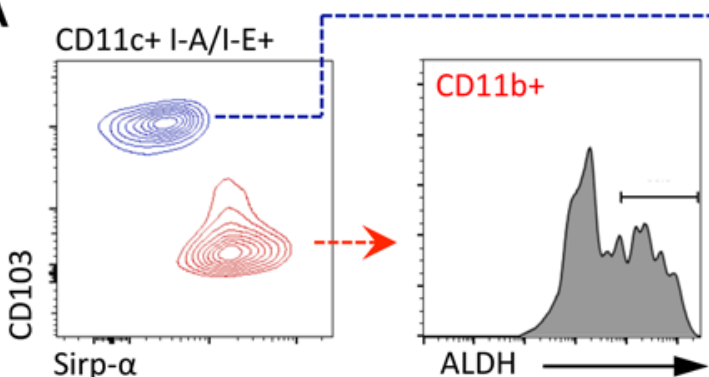

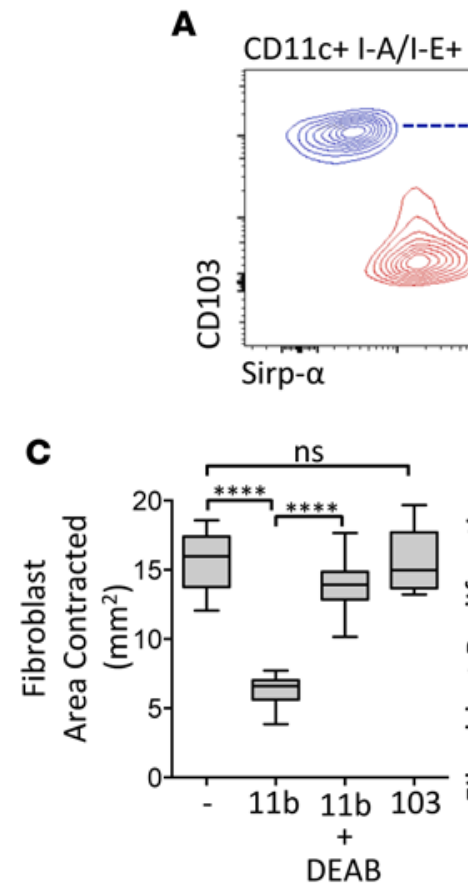

E

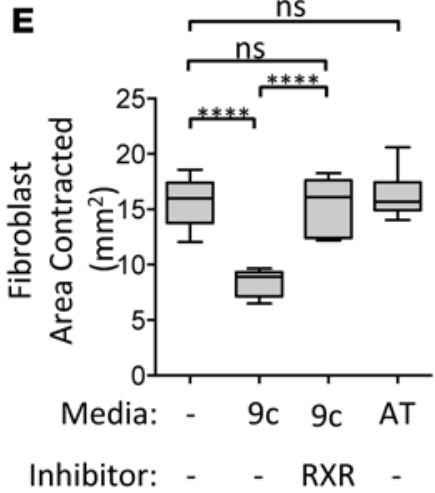

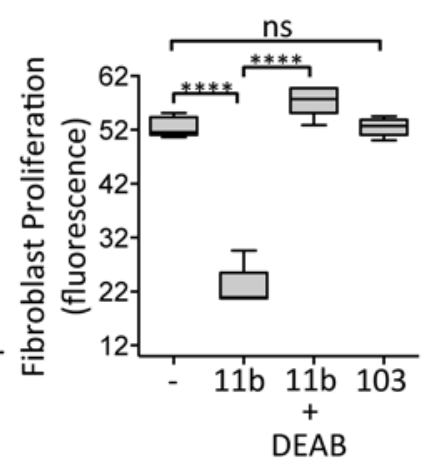

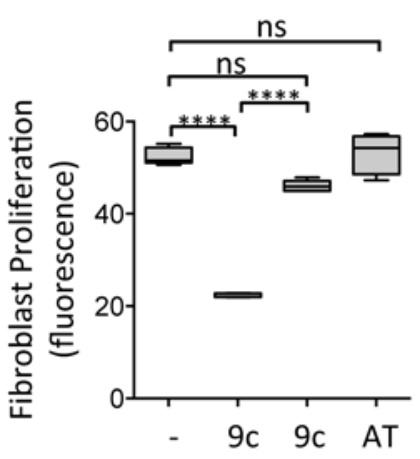

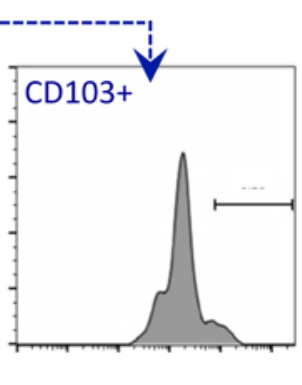
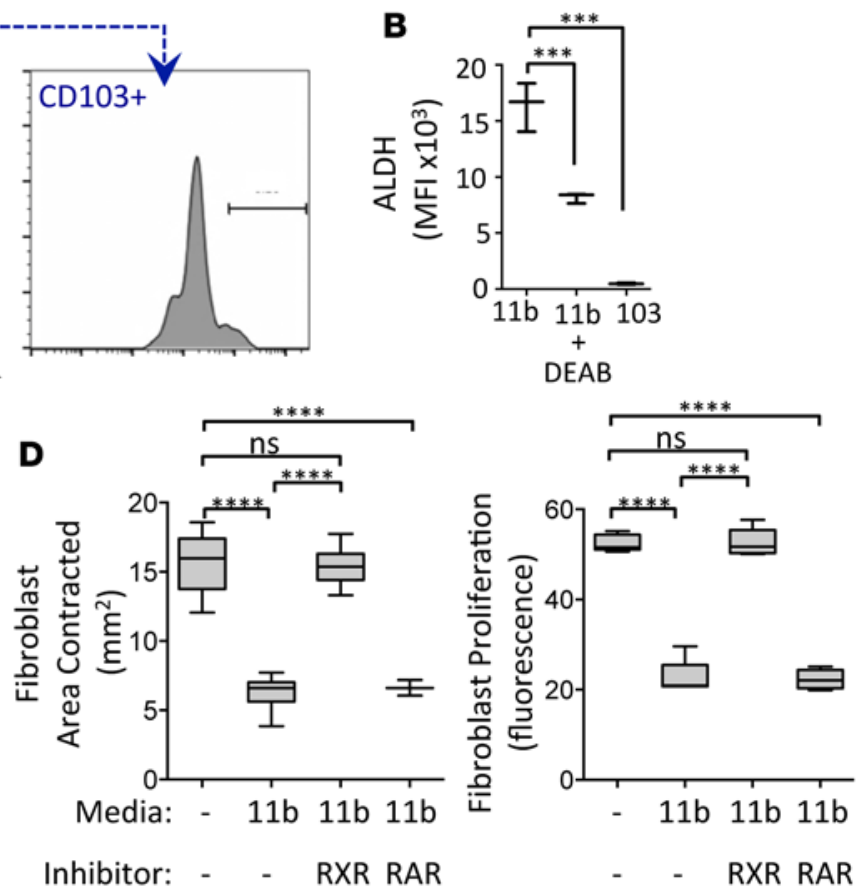

$\mathbf{F}$
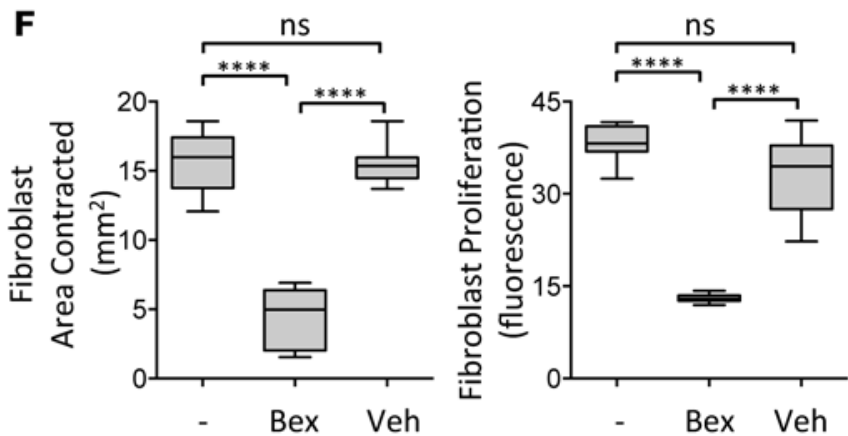

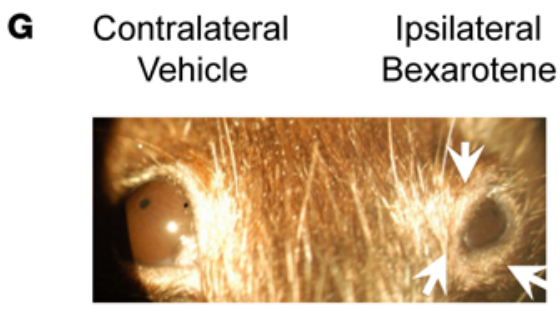

$$
\text { - } \quad \text { RXR - }
$$

\section{Contralateral Vehicle}

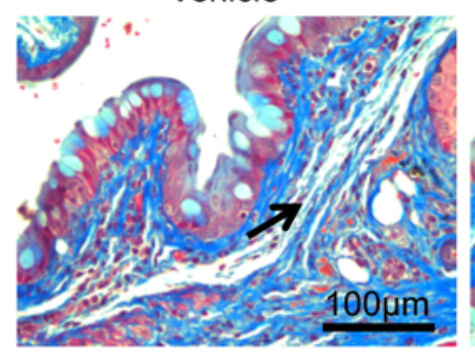

Ipsilateral

Bexarotene

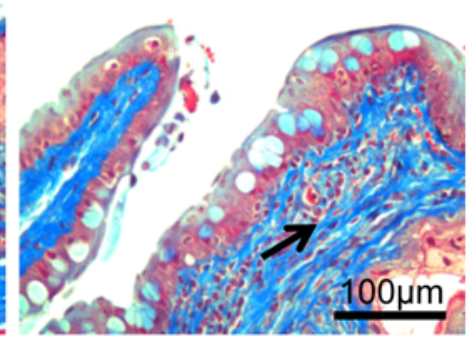

Figure 2. Fibrogenic role for CD11 b+ BMDC-derived ALDH on ocular mucosal fibroblasts. (A and B) Uniquely high aldehyde dehydrogenase (ALDH) activity of $\mathrm{CD11}^{+}$(11b) BM-derived DCs (BMDC), relative to CD103+ (103) BMDCs. (C) CD11 b BMDC-derived ALDH impairs normal fibroblast proliferation and contractility in vitro. Primary conjunctival fibroblasts were plated in media for the proliferation assay or a free-floating collagen matrix for the contractility assay. Added to the wells were complete media only, conditioned media from CD11b+ BMDCs treated with or without diethylaminobenzaldehyde (DEAB), or conditioned media from $\mathrm{CD}_{103^{+}} \mathrm{BMDC} s$ as indicated. Gel contraction and proliferation was measured $1 \mathrm{~d}$ later. (D) Retinoid $\mathrm{x}$ receptor (RXR) signaling is responsible for CD11b+ BMDC-derived ALDH impairment of normal fibroblast contractility and proliferation. Fibroblasts were plated in the presence of CD11b+ BMDC-conditioned media or not and treated with RXR or retinoic acid receptor (RAR) inhibitor as indicated. (E) 9-cis-RA (9c) mediates RXR signaling that causes impairment of normal fibroblast contractility and proliferation. Primary conjunctival fibroblasts were plated, and some samples received 9c or all-trans-RA (AT), in addition to RXR inhibitor as indicated. (F) RXR agonist, bexarotene (Bex), impairs normal fibroblast contractility and proliferation (Veh, vehicle). (C) Local administration of Bex in vivo induces ocular mucosal fibrosis. Subconjunctival injection of Bex $(10 \mathrm{ng})$ was administered to one eye, whereas the vehicle control was injected into the fellow eye. Clinical images were obtained, and Masson's trichrome-stained sections of the conjunctiva were assessed (white arrows indicate diseased eye; black arrows indicate subepithelial collagen deposition). Triplicate data points represent fibroblast data from 3 separate fibroblast cultures from 3 different mice (error bars represent $\pm S E M,{ }^{* * *} P<$ $0.0005,{ }^{* * *} P<0.00005$, 1-way ANOVA with Bonferroni correction). In vivo data are representative of $n=5$ mice. Each experiment was carried out at least twice. 
intra- and extracellular collagen production but lower levels of contraction and proliferation compared with those of normal controls. The central role of ALDH in this modulatory effect was confirmed in our study by application of conditioned media from DEAB-treated CD $11 b^{+}$BMDCs, which prevented decreased fibroblast proliferation and contraction (Figure $2 \mathrm{C}$ ). In addition, consistent with our finding that $\mathrm{CDC} 1$ have low-level ALDH activity, use of $\mathrm{CD} 103^{+} \mathrm{BMDC}$-conditioned media did not alter normal fibroblast proliferation and contraction levels (Figure 2C). Taken together, our data suggest a possible fibrogenic role for $\mathrm{CD} 11 \mathrm{~b}^{+} \mathrm{BMDC}$-derived ALDH on primary conjunctival fibroblasts.

To further characterize the mechanism by which $C D 11 b^{+}$BMDC-derived ALDH mediates fibrogenesis, we evaluated the role of RAR and RXR on fibroblasts, i.e. nuclear receptors in RA signaling. We accomplished this aim by assessing the effect of RAR or RXR inhibitors on primary conjunctival fibroblasts cultured in fibrogenic CD11b $\mathrm{b}^{+} \mathrm{BMDC}$-conditioned media. Data show that such conditioned media were no longer fibrogenic in the presence of RXR inhibitor, as fibroblast contraction and proliferation levels were normal (Figure 2D). This restorative effect was not observed with RAR inhibition (Figure 2D). These data suggest that $\mathrm{CD} 11 \mathrm{~b}^{+} \mathrm{BMDC}$-derived ALDH mediates fibrogenesis through RXR and thereby prompted us to next examine whether RXR ligands 9-CisRA or ATRA are likewise fibrogenic. Indeed, we found that addition of 9-CisRA to primary conjunctival fibroblasts led to decreased contraction and proliferation (Figure 2E), and this effect was abolished when 9-CisRA was added with RXR inhibitor (Figure 2E). In contrast, ATRA did not exhibit this effect (Figure 2E). We also asked whether agonizing RXR on primary conjunctival fibroblasts is likewise fibrogenic. Indeed, data show that the addition of RXR agonist bexarotene is fibrogenic, as reduced contraction and proliferation levels in fibroblasts were observed (Figure 2F). Thus, our data together suggest that ALDH from CD11b+ BMDCs mediates fibrogenesis via activation of $\mathrm{RXR}$ in conjunctival fibroblasts.

We further went on to assess the role of this RXR agonist in vivo and did so via subconjunctival injection of bexarotene $(0.2 \mathrm{ng} / \mu \mathrm{l})$ into one eye of naive mice (i.e., ipsilateral eye) while the fellow (i.e., contralateral) eye was given the vehicle control. Data showed that bexarotene indeed led to fibrosis, as indicated clinically and histologically (Figure 2G). The vehicle-treated control eye was unaffected (Figure 2G). Thus, our data indicate that RXR activation causes ocular mucosal fibrosis in vivo and also corroborate that reduced proliferation and contraction of conjunctival fibroblasts in vitro reflects a profibrotic state.

Association of ALDH activity by $c D C 2$ s with ocular mucosal fibrosis during allergic inflammation. The fibrogenic properties of ALDH activity produced by $\mathrm{CD} 11 \mathrm{~b}^{+}$BMDCs prompted us to next ask whether these cells are also profibrotic in vivo in the ocular mucosa. We addressed this question by taking advantage of an established mouse model of AED (30, 33, 34), which involves systemic OVA immunization and followed 2 weeks later with once/day OVA eye drop instillations for 7 consecutive challenge days. This model is relevant because the human form of this condition (i.e., atopic keratoconjunctivitis) causes scarring of the ocular mucosa (27). Similarly, we found that mice with AED indeed developed mucosal fibrosis, evidenced both clinically and histologically (Figure 3, A and B). Furthermore, confocal microscopy of ocular mucosal whole-mounts revealed that fibrosis was concurrent with increased ALDH activity (Figure 3C) and that ALDH was colocalized with antigen presenting cells (I-A/I-E $\mathrm{E}^{+}$(Figure 3C).

These findings prompted us to examine the status of ALDH activity in ocular mucosal cDC2s during AED, which we accomplished via flow cytometry (Figure 3, D and E). Tissues were harvested at baseline and then at 20 minutes and 6 hours after challenge on each day. Consistent with our observations in the steady state, $\mathrm{cDC} 2$ conferred the highest ALDH activity, both at the level of the ocular mucosa and LN (Figure 3F). Just as striking, we observed that ALDH activity in these cells increased significantly on day 2 (at 6 hours after challenge) and on day 3 (at 20 minutes and 6 hours after challenge) (Figure 3G). Comparatively, other myeloid cells showed lower levels of ALDH activity, both in the ocular mucosa and draining LN (Supplemental Figure 3). Furthermore, enumeration of $\mathrm{cDC} 2$ revealed that these cells increased in number, as well (Figure 3E). Thus, our data allowed us to conclude that an increase in the number of cDC2s occurs in AED and that their ALDH activity increases on a per-cell basis.

Local Inhibition of $c D C 2$-derived ALDH protects mice from ocular mucosal fibrosis during allergic inflammation. Based on our in vitro and in vivo data, we next sought to address whether $\mathrm{cDC} 2$-derived ALDH contributes to mucosal fibrosis in AED. Our approach was to treat AED mice topically with the ALDH inhibitor DEAB once per day in one eye, whereas the contralateral eye was instead treated with the vehicle (Figure 4A). In this way, the contralateral eye serves as an internal control. More importantly, secondary activation of $\mathrm{T}$ cells in the draining LN of the untreated/contralateral eye can occur, which in turn ought to permit these contralateral T 
A

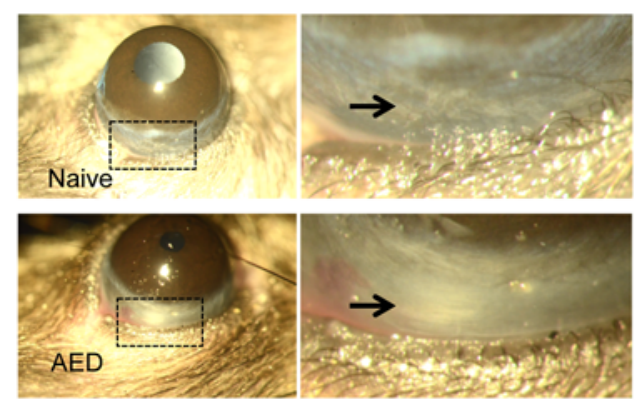

B
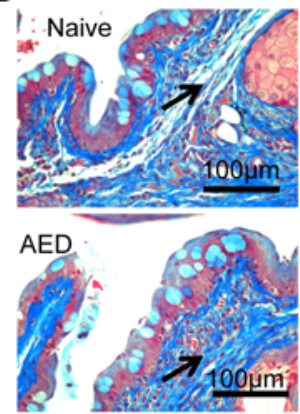

C
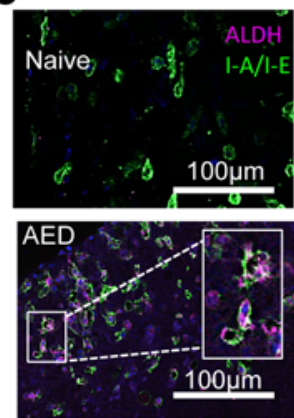

D Oc Mucosa
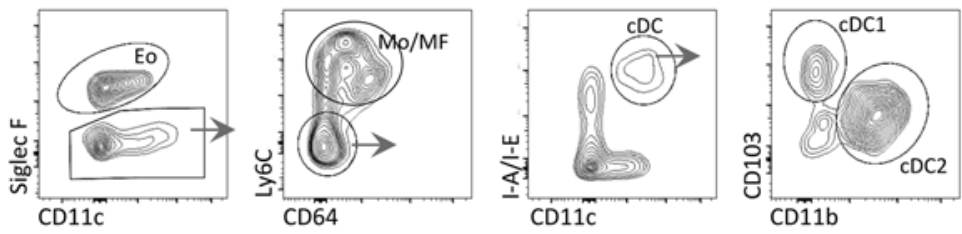

E LN (Cervical/Submandibular)
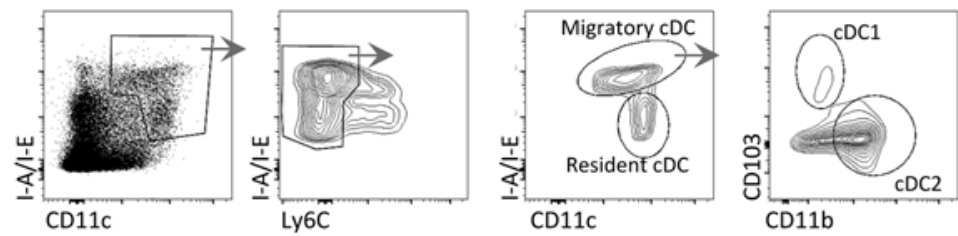

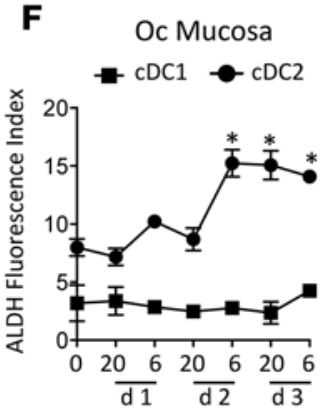

Challenge Day
Lymph Node

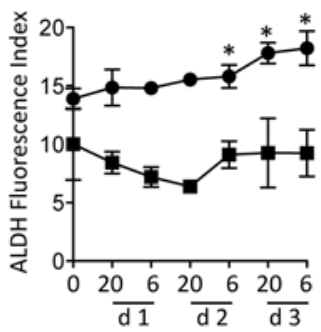

Challenge Day
G

Oc Mucosa

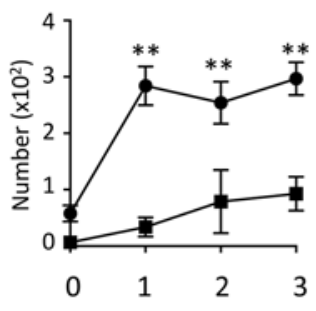

Challenge Day

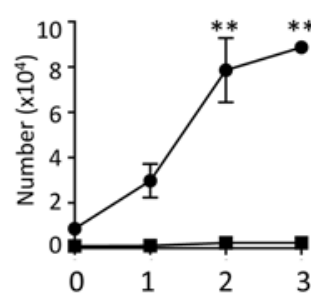

Challenge Day

Figure 3. Development of ocular mucosal fibrosis during allergic eye disease (AED) is associated with augmented cDC2-derived ALDH activity. Mice were immunized with OVA/adjuvant and challenged topically once daily with an OVA instillation to induce AED. (A and B) Ocular mucosal fibrosis develops in AED (black arrows). Data from whole eyes sectioned and stained with Masson's trichrome are representative of $n=3$ per group (inset shows fibrosis/opaque white lesion in AED, not seen in naive mice). (C-F) Ocular (Oc) mucosal type-2 classical DC (CDC2) confer increased aldehyde dehydrogenase (ALDH) activity in AED. (C) Whole mount immunofluorescence of the ocular mucosa is representative of $n=5$ per group. ( $\mathbf{D}$ and $\mathbf{E}$ ) Ocular mucosa and draining lymph node (LN) in AED were collected for flow cytometry analysis. Representative flow cytometry plots are shown from tissues collected at $6 \mathrm{hr}$ after challenge on day 3. (F) Quantitation was performed on tissues collected at $20 \mathrm{~min}$ (20) and $6 \mathrm{hr}$ (6) after OVA challenge on days (d) 0 through 3 to measure ALDH activity by flow cytometry. (G) Ocular mucosal CDC2 increase in number during AED. Flow cytometry was carried out for enumeration of cDCs. Data shown for the ocular mucosal cDCs are representative of duplicate data points from 2 separate experiments. Cell suspensions were pooled samples from $n=10$ naive mice or $n=6$ mice for each experiment (error bars represent $\pm \mathrm{SEM} ;{ }^{*} P<0.05,{ }^{*} P<0.005$; 1 -way ANOVA with Bonferroni correction). Each experiment was carried out at least twice.

cells to induce AED equivalently in both eyes, as was demonstrated in Figure 4A. The cDC2s in DEAB-treated eyes showed significantly reduced ALDH activity, whereas the vehicle-treated/contralateral eye maintained normal levels (Figure 4B). Thus, unilateral DEAB administration inhibits CDC2-derived ALDH in the treated eye only, while both eyes experience equivalent and fulminant levels of allergic inflammation. 
A

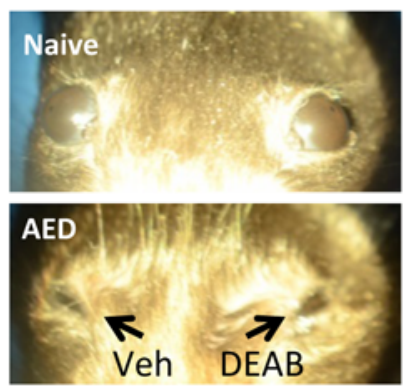

C
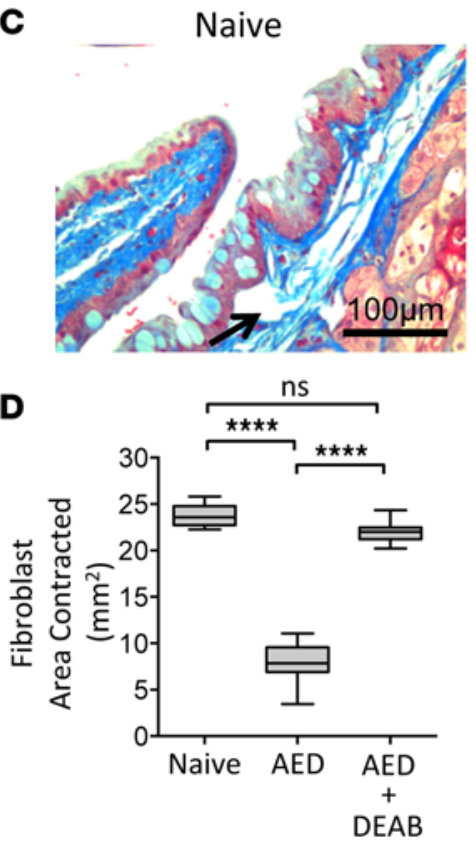

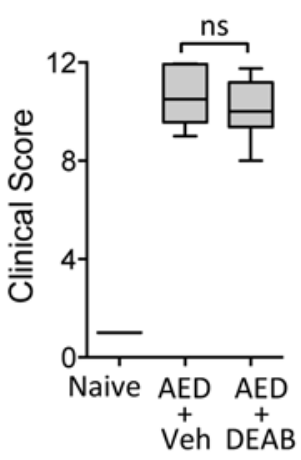

AED + Vehicle
B

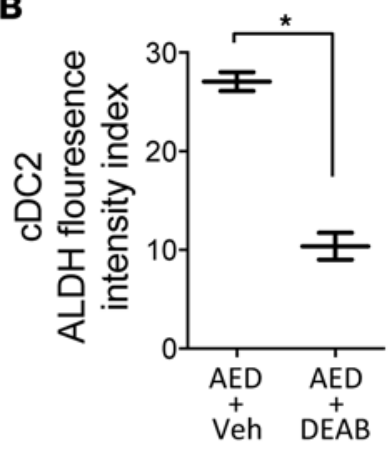

$\mathbf{E}$
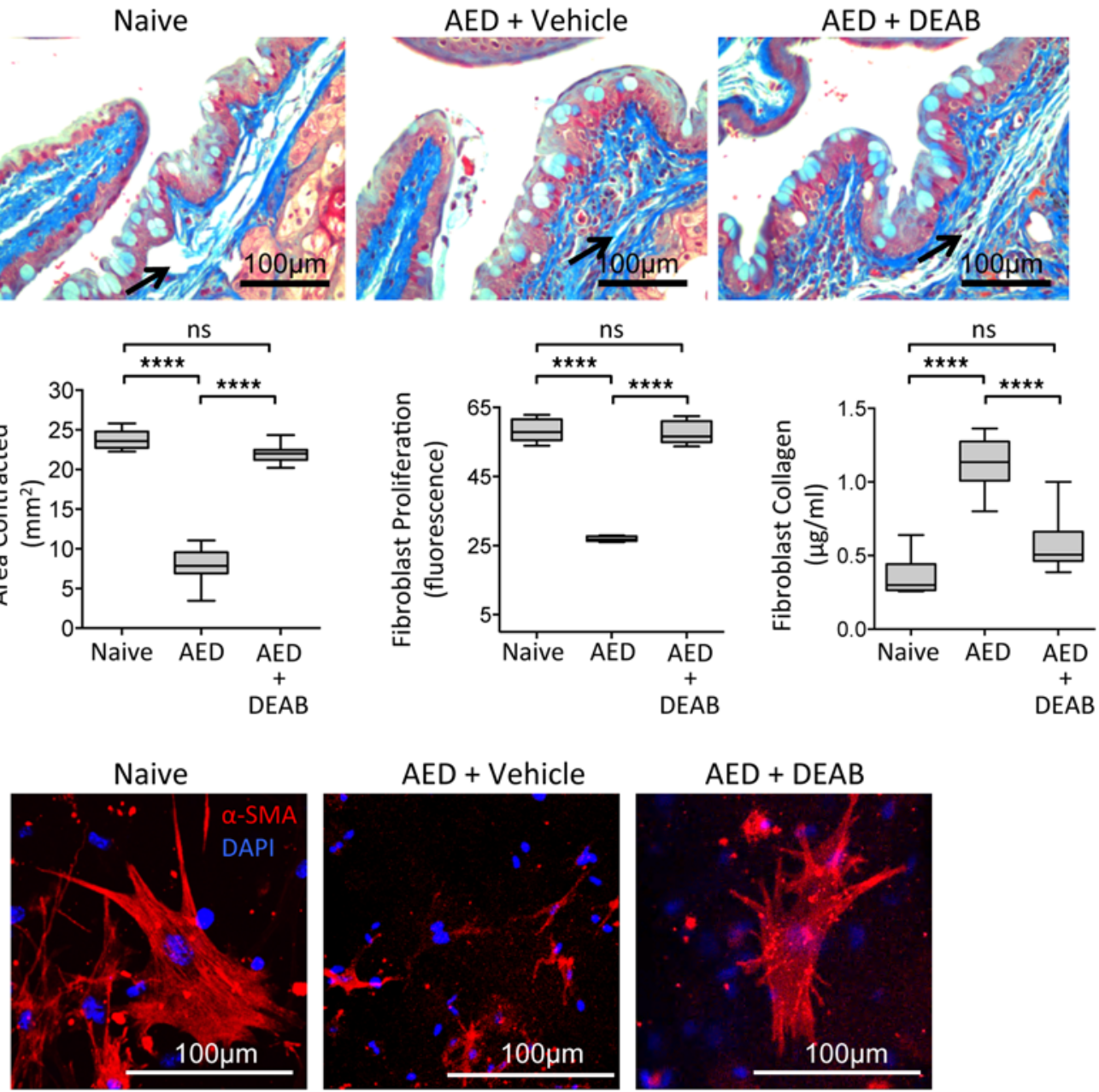

Figure 4. Local inhibition of CDC2-derived aldehyde dehydrogenase (ALDH) protects mice from ocular mucosal fibrosis during allergic inflammation. Mice induced with allergic eye disease (AED) were treated with topical diethylaminobenzaldehyde (DEAB) in one eye and vehicle control in the fellow/contralateral eye. (A and B) Equivalent levels of allergic inflammation in AED is achieved in the setting of unilateral DEAB instillation (black arrows). Clinical image and scores are derived from $n=12$ mice. (B) Unilateral DEAB instillation in AED leads to reduced ALDH activity in type-2 classical DC (CDC2), as compared with those from the vehicle-treated contralateral eye. Flow cytometry data are derived from triplicate data points of pooled conjunctiva from $n=12$ mice. (C) Unilateral DEAB instillation during AED leads to reduced collagen deposition in the ocular mucosa of the treated eye (black arrows). Images represent $n=6$ mice. ( $\mathbf{D}$ and $\mathbf{E}$ ) Unilateral DEAB instillation restores normal levels of proliferation, contractility, collagen production, and $\alpha$ smooth muscle actin $(\alpha)$ of AED conjunctival fibroblasts from the treated eye. Fibroblast data are represented by triplicate data points from 3 separate fibroblast cultures from 3 different mice (error bars represent \pm SEM; ${ }^{*} P<0.05,{ }^{* * *} P<0.00005$; 1 -way ANOVA with Bonferroni correction). Each experiment was carried out at least twice. 
Using this approach, we next looked at Masson's trichrome staining and found that impairing conjunctival cDC2-derived ALDH reduced subepithelial collagen deposition in the ocular mucosa during AED (Figure 4C). We also cultured primary conjunctival fibroblasts from DEAB- and vehicle-treated eyes from these mice. Data showed that, compared with vehicle-treated controls, fibroblasts from the DEAB-treated eyes were restored with normal levels of contraction, proliferation, collagen production, and $\alpha$ smooth muscle actin ( $\alpha$-SMA) expression (Figure 4, D and E). Thus, our data suggested that cDC2-derived ALDH causes ocular mucosal fibrosis during allergic inflammation.

Local depletion of ocular mucosal cDCs protects mice from developing fibrosis during allergic inflammation. Our finding that targeting $\mathrm{CDC} 2$-derived ALDH protects mice from ocular mucosal fibrosis in AED would therefore predict that the depletion of cDCs ought to have a similar protective effect. To address this possibility, we took advantage of CD11c-eGFP/DTR mice and deleted CD11 $\mathrm{c}^{+}$cells by topical application of diphtheria toxin (DTx) during AED. Similar to the previous experiment, DTx was administered only to the ipsilateral eye (on days $-1,3$, and 5), while the contralateral eye received a vehicle as an internal control. In this way, secondary activation of $\mathrm{T}$ cells can occur in the draining $\mathrm{LN}$ of the untreated/contralateral eye, which in turn ought to permit these contralateral T cells to induce AED equivalently in both eyes. Indeed, AED levels developed equivalently in both eyes (Figure 5A). We also verified using intravital multiphoton microscopy in this setting that our approach only depleted DCs in the ipsilateral DTx-treated eye (Figure 5B). Consistent with this finding, we found in the AED setting that the ipsilateral draining LN likewise had reduced migratory cDC2s (Figure 5B and Supplemental Figure 4). A reduction of monocyte-derived (Mo-derived) DCs in the ipsilateral LN was also observed; however, lymphoid-resident cDCs were not affected (Figure 5B and Supplemental Figure 3).

Using this approach we found that DC depletion resulted in reduced subepithelial collagen deposition in the AED setting, whereas the contralateral control eye did not (Figure 5C). These data were corroborated using primary conjunctival fibroblasts from such DC-deficient eyes, as primary conjunctival fibroblasts were restored with normal levels of contraction, proliferation, and collagen production (Figure 5D). Thus, our data together allowed us to conclude that depletion of DCs reduces levels of ocular mucosal fibrosis during allergic inflammation.

\section{Discussion}

We herein establish that $\mathrm{cDC} 2 \mathrm{~s}$ in mice mediate ocular mucosal fibrosis in allergic inflammation, which is the first demonstration that DCs directly contribute to fibrosis. Our findings add to previous studies that have associated DCs with fibrosis indirectly, i.e., via their recruitment/activation of other profibrotic leukocytes $(1-3,35)$. The latter is exemplified by elegant work from Connolly et al., who recently reported that DC production of TNF- $\alpha$ governs hepatic inflammation that causes liver fibrosis in mice (36).

Additionally, Kitamura et al. associated DCs and inflammation with $\alpha v \beta 8$-mediated activation of latent TGF- $\beta$ by fibroblasts in pulmonary fibrosis (37). Although indeed important, these are examples of indirect pathways for DC-mediated fibrosis, whereas we have demonstrated that $\mathrm{CDC}$ can mediate fibrosis directly in the ocular mucosa, via RA synthesis. We also definitively show it is the CDC2s that mediate this effect, and these cells do so through their ALDH activity, which is augmented in allergic inflammation. As our data suggest, fibrogenesis in this setting occurs via ligation of RA by conjunctival fibroblast RXR

Pursuit of our investigation was followed by Dart and colleagues' findings of ALDH-mediated autoregulation of a profibrotic phenotype in conjunctival fibroblasts from patients with MMP (29). Their results led us to ask whether $\mathrm{cDC} 2 \mathrm{~s}$ also had fibrogenic potential in the ocular mucosa, given our observations that these cDCs harbored significant ALDH activity in steady-state ocular mucosa and in draining LN. Interestingly, we also found a similar trend in steady-state cDC2s in the lung, as well as in CD11b+ BMDCs, reported also by Yokota et al. (25). Furthermore, our data agree with Guilliams et al., who demonstrated an increased frequency of $\mathrm{ALDH}^{+}$cells in dermal $\mathrm{CD}^{-103^{-}} \mathrm{cDCs}$ as compared with $\mathrm{CD} 103^{+}$counterparts (24). Likewise, we were able to demonstrate, with transcriptome data generated by Heng et al. of the Immunological Genome Project Consortium, a similar trend with Aldh1a2 mRNA levels in CD11b ${ }^{+}$vs. $\mathrm{CD}_{103^{+}}$cDCs from skin-draining LN (i.e., MHCII ${ }^{\text {hi }}$ Langerin ${ }^{-} \mathrm{CD} 103^{-} \mathrm{CD}^{-11 b^{+}}$vs. $\mathrm{MHCII}^{\text {hi }}$ Langerin $^{+} \mathrm{C}-$ $\mathrm{D}_{103}{ }^{+} \mathrm{CD} 11 \mathrm{~b}^{-} \mathrm{cDCs}$, respectively), liver and lung (CD103-CD $11 \mathrm{~b}^{+} \mathrm{CD} 24^{+}$vs. $\left.\mathrm{CD} 103^{+} \mathrm{CD} 11 \mathrm{~b}^{-}\right)$, and mediastinal LN (26). Thus, it is tempting to hypothesize that high ALDH activity and associated fibrogenic potential may be a unique feature intrinsic to the $\mathrm{CDC} 2$ cluster (28), which may predict high ALDH activity in the recently described cDC2-commited progenitor (38). However, we show that lymphoid-resident 
A

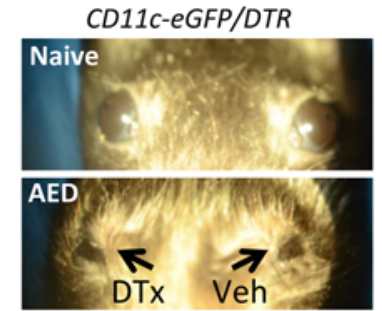

B

Oc Mucosa
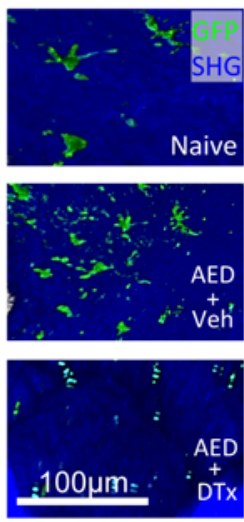

C

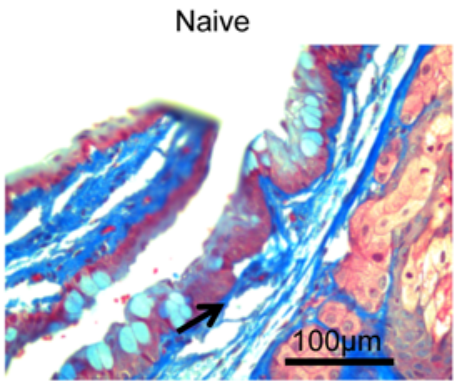

D

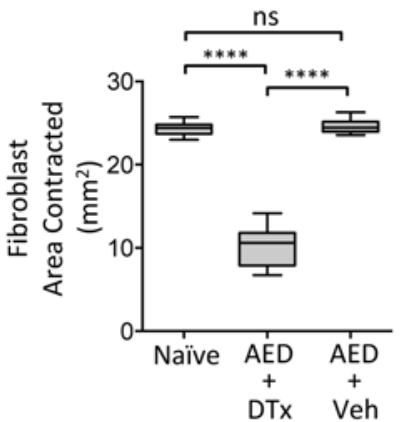

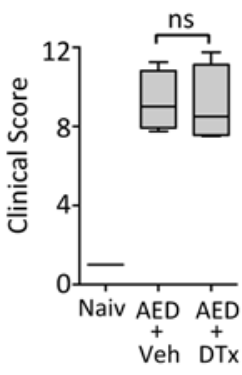

Migratory $\mathrm{CDC}$ (CD11 $c^{\text {int }}$ I-A/E ${ }^{\text {hi}}$ )
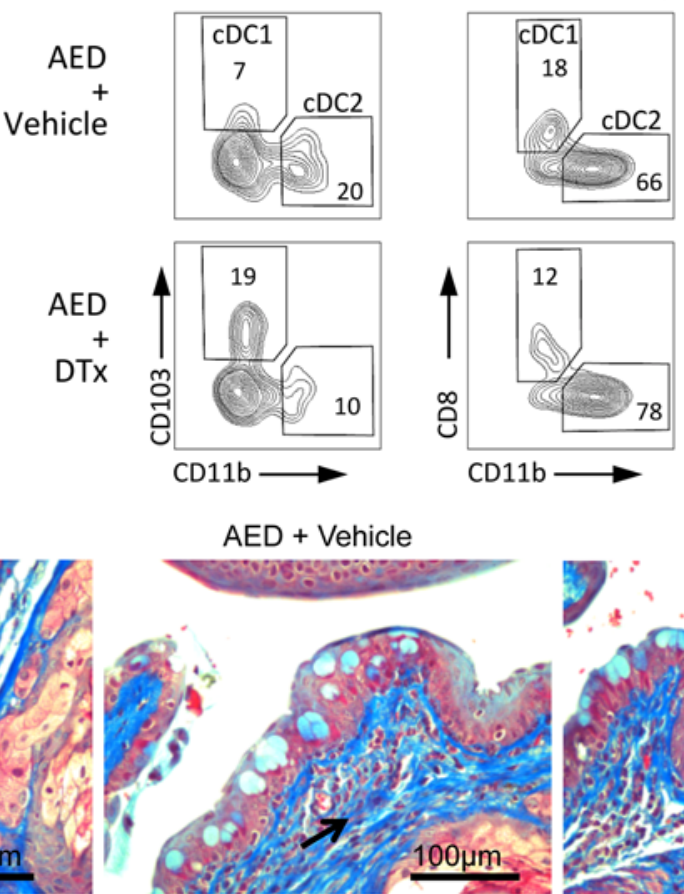

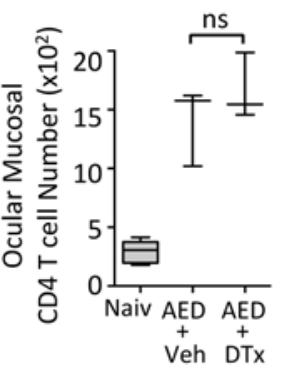

Mo DC $\left(\mathrm{CD} 11 \mathrm{c}^{+} \mathrm{I}-\mathrm{A} / \mathrm{E}^{+}\right.$Ly6C $\left.\mathrm{C}^{+}\right)$
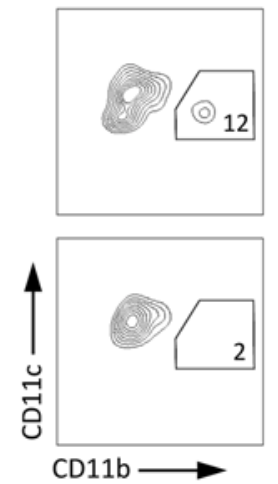

AED + DTx
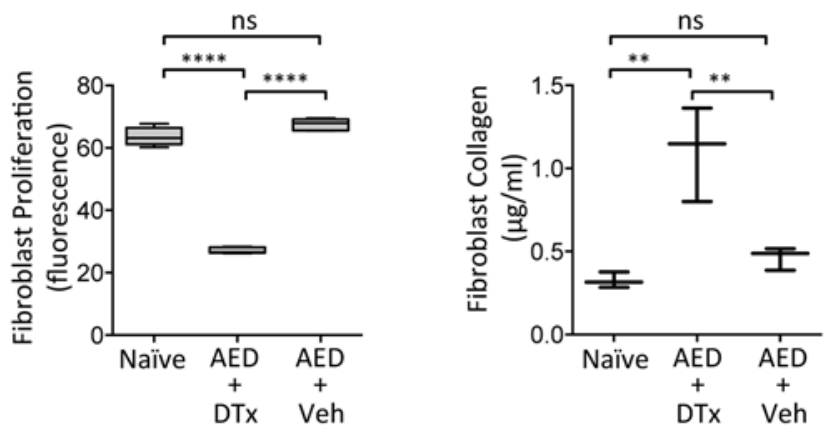

Figure 5. Local DC depletion protects CD11c-eGFP/DTR mice from developing ocular mucosal fibrosis during allergic inflammation. Ipsilateral diphtheria toxin (DTx) instillation was given topically ( $3.125 \mathrm{ng}$ ) on days $-1,3$, and 5 of challenge, whereas the contralateral eye received PBS control. (A) Equivalent levels of allergic inflammation in allergic eye disease (AED) is achieved despite unilateral DTx instillation to CD11c-eGFP/DTR mice (black arrows). Clinical scores and enumeration of ocular mucosal eosinophils (Eos) and CD4+ T cells (flow cytometry) compared DTx-treated eyes vs. PBS-treated contralateral eyes on day 7. (B) Deletion efficacy of unilateral DTx instillation. Intravital multiphoton microscopy of DTx-treated eye and vehicle-treated contralateral eyes was carried out in AED mice to verify DC deletion in the DTx-treated eye (SHG, second harmonic generation). Lymph nodes (LNs) were collected for flow cytometry to verify deletion of ipsilateral migratory type-2 classical DCs (CDC2). Data is derived from triplicate data points from $n=5$ mice. (C) Unilateral DTx instillation during AED leads to reduced collagen deposition in the ocular mucosa of the treated eye (black arrows). Micrographs represent $n=5$ mice. (D) Unilateral DTx instillation restores normal levels of proliferation, contractility, and collagen production of AED conjunctival fibroblasts from the treated eye. Fibroblast data are represented by triplicate data points from 3 separate fibroblast cultures from 3 different mice (error bars represent \pm SEM; ${ }^{* *} P<0.005,{ }^{* * *} P<0.00005$; 1-way ANOVA with Bonferroni correction). Each experiment was carried out at least twice. 
cDC2s in the submandibular/cervical LN do not appear to harbor ALDH activity. Also known to express high levels of ALDH are cDC1s in the small intestine lamina propria. Thus, high ALDH activity in $\mathrm{cDC} 2 \mathrm{~s}$ cannot solely be an intrinsic quality of their particular developmental lineage, but it ought to also be an effect of certain tissue-specific factors.

We identified the likely mechanism by which cDC2-derived ALDH activity mediates ocular mucosal fibrosis. First, we demonstrated using primary conjunctival fibroblasts that ALDH produced by CD11 ${ }^{+}$ BMDCs is fibrogenic in vitro, since conditioned media from DEAB-treated CD $11 b^{+}$BMDCs prevented this result. Second, and supportive of this profibrotic role for $\mathrm{CD} 11 \mathrm{~b}^{+} \mathrm{BMDC}$-derived ALDH, we proved that addition of 9-CisRA caused the same fibrogenic effect. This finding is in agreement with Okuno et al., who observed a similar result of adding 9-CisRA to rat hepatic stellar cells (7). Third, we demonstrated with agonist/antagonist experiments on primary conjunctival fibroblasts that RXR signaling mediates the observed fibrogenic effect in this system. These findings support our aforementioned observation that 9-CisRA is fibrogenic, since this isomer is the high-affinity ligand of $\operatorname{RXR}(22,23)$. Importantly, we were able to show in vivo that agonizing RXR via bexarotene injection in vivo led to ocular mucosal fibrosis. Thus, our data collectively suggest that cDC2-derived ALDH plays a key role in consequent 9-CisRA synthesis, which is fibrogenic to the ocular mucosa.

In vivo evidence that $\mathrm{cDC} 2$-derived $\mathrm{ALDH}$ activity is central to mediating ocular mucosal fibrosis in the AED setting was also obtained. We showed that the absolute numbers of ocular mucosal $\mathrm{cDC} 2 \mathrm{~s}$ increase significantly in AED, which agrees with the same increase in $\mathrm{CD} 11 \mathrm{~b}^{+} \mathrm{cDC}$ reported by Plantinga et al. in the allergic airway inflammation setting (39). We also show that ocular mucosal cDC2s increase their ALDH activity significantly in AED, and thus one can surmise that both their increased ALDH activity and absolute numbers could result in enhanced levels of profibrotic 9-CisRA in the AED. Of note, in our study, no other myeloid population examined (e.g., Mos, eosinophils, macrophages, or PMN) showed persistent levels of ALDH activity similar to that of ocular mucosal cDC2s. Likewise, transcriptome data from Heng et al. corroborate low Aldh1a2 mRNA levels in granulocytes, Mos, and macrophages (except for peritoneal macrophages), as well as in lymphocytes and innate lymphoid cells (26), suggesting that high activity of ALDH1 enzymes is not conserved across all immune cell types, as previously appreciated (19).

Our key in vivo findings came from experiments in which we were able to perturb cDCs in the AED setting. By taking advantage of a similar technique shown by Gao et al. to deplete ocular DCs through local DTx administration in CD11c-eGFP/DTR mice (40), we were able to demonstrate that DC depletion in the ocular mucosa resulted in significantly reduced levels of fibrosis in the AED setting. Interestingly, Jiao et al. recently reported that DCs and plasmacytoid DCs (pDCs) contribute to regression of liver fibrosis in the carbon-tetrachloride model and that systemic DTx administration in CD11c-eGFP/DTR mice led to a delay in this regression (41). In our study, we further verified the contribution of cDC2-derived ALDH activity in ocular mucosal fibrosis via topical administration of DEAB, which led to significantly reduced levels of fibrosis during AED. Thus, consistent with our in vitro findings, our data collectively suggest that ALDH activity from $\mathrm{cDC} 2 \mathrm{~s}$ is responsible for ocular mucosal fibrosis during AED.

Importantly, this in vivo approach in testing the role of cDC2-derived ALDH was carefully devised so as to manipulate DCs without reducing allergic inflammation. Uncoupling inflammation and fibrosis is particularly important when studying RA, as retinoids can suppress fibrogenic inflammation $(7,19,24,42)$. In the allergy setting, DCs trigger secondary Th2 responses that perpetuate allergic inflammation, as well; therefore, reducing $\mathrm{T}$ cell responses could also lessen fibrogenic inflammation $(1-3,35)$. Thus, to study ocular mucosal fibrosis while maintaining fulminant allergic inflammation levels, we induced AED in both eyes but only manipulated ocular mucosal cDCs unilaterally. In this way, the control eye would drive full development of secondary Th2 responses systemically to elicit fulminant AED in both eyes. The data supported our hypothesis, since ipsilateral DEAB treatment in one series of experiments and ipsilateral DTx treatment in the other experiments resulted in equivalent AED and cellular recruitment levels in both eyes. Thus, we were able to dissect out the direct fibrogenic role of cDC2-derived ALDH in the AED setting.

The studies described in the accompanying paper, from Dart and colleagues, and our work here, both converge on the finding that ALDH/RA plays a central role in ocular mucosal fibrosis in mice and humans (29). Their study provides evidence that the ALDH/RA pathway is critical in conjunctival fibrosis seen in MMP patients and further shows, in the same AED mouse model used here, that conjunctival scarring also develops . Sensitized mouse conjunctival fibroblasts were demonstrated in their study to behave identically to human diseased fibroblasts in vitro, with respect to their profibrotic profile and restoration of normal 
functionality by in vivo topical application of ALDH inhibitor disulfiram during AED. Our work demonstrates a paracrine profibrotic effect of ALDH/RA on conjunctival fibroblasts produced by $\mathrm{cDC} 2 \mathrm{~s}$ in AED and may suggest a similar role for the human $\mathrm{CD} 1 \mathrm{c}^{+} \mathrm{CDC}$ homologue (reviewed in ref. 43), whereas Dart and colleagues' work highlights an autocrine profibrotic mechanism in ALDH/RA by conjunctival fibroblasts in human ocular MMP and in mouse AED (29). Their conclusion in human MMP was supported by upregulated ALDH found in conjunctival fibroblasts from these patients and by the fact that these fibroblasts maintain a range of profibrotic characteristics in vitro that are restored to normal by application of ALDH inhibitors. Thus, their findings identify this pathway as an underlying mechanism in the previously unknown pathogenesis of progressive conjunctival scarring.

In summary, we herein demonstrate that $\mathrm{cDC} 2 \mathrm{~s}$ are inducers of ocular mucosal fibrosis in the allergic setting, which is the first in vivo demonstration that DCs can directly effect fibrosis. Work in the companion study shows ALDH-mediated autoregulation of a profibrotic phenotype in conjunctival fibroblasts from patients with MMP (29). We show that uniquely high ALDH activity of cDC2s is fibrogenic in allergic inflammation, which our data suggest leads to ligation of 9-CisRA by RXR on conjunctival fibroblasts. Our findings not only have the potential to open up a novel area for fibrosis research in CDC-mediated retinoid metabolism, but also advance the current understanding of the DC-RA axis beyond its already appreciated role in T cell homing and tolerance. Future work is required to determine whether cDCs in humans likewise mediate fibrosis of the ocular mucosa in allergy and whether our findings also extend to other fibrosis in other etiologies, such as trachoma.

\section{Methods}

Animals and euthanasia. Female C57BL/6 (B6) mice aged 8-12 weeks were purchased from Charles River Laboratories. CD11c-eGFP/DTR mice also 8-12 weeks were gifted from the labs of Soman Abraham (Department of Pathology, Duke University) and Mari Shinohara (Department of Immunology, Duke University), which were originally purchased from Jackson Laboratory (stock no. 004509). Mice were housed in a specific pathogen-free environment at the Duke Eye Center animal facility. Anesthesia was used for all surgical procedures with i.p.-administered ketamine/xylazine suspensions (120 and $20 \mathrm{mg} / \mathrm{kg}$, respectively).

AED. AED was induced in 9-12 week old WT B6 mice, as previously described $(30,33,34)$. Briefly, one i.p. administration of ovalbumin (OVA; $50 \mathrm{ng} / \mu 1$, Sigma-Aldrich) emulsified in aluminium hydroxide $(2 \mu \mathrm{g} / \mu 1$, Thermo Scientific) and pertussis toxin $(1.5 \mathrm{ng} / \mu 1$, Sigma-Aldrich), in a total volume of $200 \mu 1$ of sterile saline. Two weeks later, mice received a daily eye drop instillation of $5 \mu \mathrm{l}$ OVA $(50 \mu \mathrm{g} / \mu \mathrm{l})$ in sterile saline for 7 days. Clinical parameters including eyelid edema, chemosis, conjunctival redness, and tearing were each scored on a scale of $0-3+$, as previously described $(30,33,34)$.

BMDCs. BMDCs were generated as previously described $(25,30,31,33$, 34). Briefly, femurs and tibiae were collected from freshly euthanized B6 mice, BM harvested, and single cell seeded at $2 \times 10^{5} / \mathrm{ml}$. RPMI 1640 (Lonza) was supplemented with 10\% FBS and 1\% penicillin/streptomycin, plus $20 \mathrm{ng} / \mathrm{ml}$ mouse GM-CSF (all from BioLegend). For generation of CD103 ${ }^{+}$BMDCs, Flt-3 (100 ng/ml, eBioscience) was used instead of GM-CSF. On day 4, fresh GM-CSF $(20 \mathrm{ng} / \mathrm{ml})$ was added to both culture conditions.

Primary conjunctival fibroblasts. Primary conjunctival fibroblasts were expanded from whole conjunctival tissue explants excised from freshly euthanized B6 mice and placed under a glass coverslip with RPMI 1640 supplemented with 10\% FBS and 1\% penicillin/streptomycin. Fibroblasts between passages 4-6 were utilized in the assays described below.

Fibroblast collagen production assay in vitro. Fibroblasts were seeded at $3 \times 10^{4}$ cells per well on a 24 -well plate for 24 hours. Collagen solubilization was carried out first with cell lysis using $0.05 \mathrm{M}$ acetic acid (Sigma-Aldrich) followed by a 2-step digestion using pepsin $(0.1 \mathrm{mg} / \mathrm{ml}$; Sigma-Aldrich) and pancreatic elastase $(0.1 \mathrm{mg} / \mathrm{ml}$; Sigma-Aldrich). Collagen was solubilized and measured using mouse collagen type 1 ELISA according to the manufacturer's protocol.

Fibroblast matrix contraction assay in vitro. Fibroblasts were plated at a density of $1.6 \times 10^{6} \mathrm{cells} / \mathrm{ml}$ with $10 \times$ concentrated MEM (Invitrogen) and collagen type 1 solution $(2 \mathrm{mg} / \mathrm{ml}$, First Link) in a final volume of $1 \mathrm{ml}$. The collagen stock solution contains $0.6 \%$ acetic acid and was neutralized with $5 \mathrm{M} \mathrm{NaOH}$ (Sigma-Aldrich) prior to addition of cells. The collagen/fibroblast mixture (60 $\mu$ l per well of a 96 -well plate) was left to set at $37^{\circ} \mathrm{C}$ for 30 minutes in $5 \% \mathrm{CO}_{2}$ in air. RPMI 1640 supplemented with $10 \% \mathrm{FBS}$, and $1 \%$ penicillin/streptomycin was added to the gels before they were released from the well edge. Gels were incubated at $37^{\circ} \mathrm{C}$ in a humidified atmosphere of $5 \% \mathrm{CO}_{2}$ in air. Images were captured on day 1 with a standard 
digital camera. Gel areas were measured and analyzed using Image J software (NIH).

Fibroblast proliferation assay in vitro. Fibroblasts $/ \mathrm{ml}\left(5 \times 10^{4}\right)$ in a total volume of $100 \mu 1$ were seeded in a 96-well plate, and proliferation was determined using the CyQuant proliferation kit (Invitrogen) as per the manufacturers' protocol.

Manipulation of $R A$ pathway in fibroblasts in vitro. RA (9-CisRA and ATRA) (Sigma-Aldrich), RXR inhibitor HX531, and RAR inhibitor BMS 195614 (both from Tocris) were dissolved in 100\% DMSO (Sigma-A1drich). They were then diluted in a sterile saline to a final concentration of $10 \mu \mathrm{M}$ (final DMSO content of $0.01 \%$ ). These solutions were added to fibroblast assays 24 hours before day- 1 readings in each assay.

Fibroblast $\alpha$-SMA expression. Expression of fibroblast $\alpha$-SMA was assessed by confocal microscopy of fibroblasts in tethered collagen type 1 gels. The gels were formed similarly to the free-floating gels in the contraction assay; however, the gels were not released from the edges of the well. After 24 hours, the gels were stained with $\alpha$-SMA antibody (Cy3 conjugated, Sigma-Aldrich) using the staining protocol recommended by the manufacturer. Confocal microscopy was carried out using the Leica SP5 (Leica Microsystems) followed by image analysis with Leica LAS AF lite software.

Bexarotene use in vivo and in vitro. Bexarotene (B-2422 Free Acid > 99\%, LC Laboratories) was initially dissolved in $50 \%$ ethanol solution at $\mathrm{pH}$ of 13 and then neutralized to a $\mathrm{pH}$ of 7.5 with $100 \%$ ethanol solution at a $\mathrm{pH}$ of 2 . The final working concentration of bexarotene was $0.2 \mu \mathrm{g} / \mathrm{ml}$, leading to a $0.3 \%$ ethanol vehicle. Subconjunctival injection were performed as previously described (30) and used to administer bexarotene $(0.2 \mu \mathrm{g} / \mathrm{ml}, 50 \mu \mathrm{l})$ or ethanol vehicle $(0.3 \%, 50 \mu \mathrm{l})$ into to naive B6 mice. For the in vitro assay, bexarotene $(0.075 \mu \mathrm{g} / \mathrm{ml}, 0.1 \%$ ethanol vehicle) was added to fibroblasts.

$D E A B$ use in vivo and in vitro use. DEAB powder (Sigma-Aldrich) was dissolved in $100 \%$ DMSO (Sigma-Aldrich) and subsequently diluted in sterile saline to a final concentration of $300 \mu \mathrm{M}$. DEAB was applied topically to one eye of mice with AED, and vehicle (0.03\% DMSO in sterile saline) was applied to the contralateral eye once a day, 1 hour prior to challenge. For in vitro assays, DEAB (100 $\mu \mathrm{M}, 0.01 \%$ DMSO in sterile saline) was added to the BMDC cultures prior to BMDC conditioned-media being applied to fibroblast assays.

DTx preparation and administration. AED was induced in CD11c-DTR/GFP mice. Twenty-four hours prior to day 1 of OVA challenge, and on days 3 and 5, DTx $(3.125 \mathrm{ng}, 5 \mu \mathrm{l})$ was added topically to the ocular surface of the ipsilateral eye while the contralateral eye received a sterile saline $(5 \mu 1)$ vehicle. The DTx and vehicle were left on the ocular surface for 1 minute and then washed 4 times with sterile saline. Multiphoton microscopy was utilized to visualize the GFP signal in each eye of live mice on days 1 and 7 of challenge.

Collagenase tissue digestion. Tissues were minced and digested in $2 \mathrm{mg} / \mathrm{ml}$ collagenase D (Roche Diagnostics) and $0.05 \mathrm{mg} / \mathrm{ml} \mathrm{DNase}$ (Roche Diagnostics) for 45 minutes at room temperature using the GentleMACS Dissociator (Miltenyi Biotec). Cell suspensions were triturated in $20 \mathrm{mM}$ EDTA, passed through a 70- $\mu \mathrm{m}$ filter (BD Falcon; BD Biosciences), and thoroughly washed.

Flow cytometry. The following antibodies or dyes were used for flow cytometry: Viability (65-0863-14, eBioscience), ALDH/ALDEFLUOR (01700, Stem Cell Technologies), F4/80 (BM8, eBioscience), CD103 (2E7, BioLegend), Ly6C (AL-21, BD Pharmingen), Ly6G (1A8, BioLegend), CD64 (X54-5/7.1, BioLegend), I-A/I-E (M5/114.15.2, BioLegend), CD11b (M1/70, BD Pharmingen), CD11c (HL3, BD Pharmingen), CD45 (30-F11, eBioscience), Siglec-F (E50-2440, BD Pharmingen), CD8 (53-6.7, BioLegend), CD3 (17A2, BioLegend), and B220 (RA3-6B2, BioLegend). Data acquired on BD Fortessa LSRII and analyzed on FloJo (Treestar Inc.)

ALDEFLUOR. Cell suspensions were treated with ALDEFLUOR (Stem cell Technologies) as per manufacturer's instructions. Briefly, $5 \mu$ of the activated ALDEFLUOR was added to the cell suspensions and then incubated in the dark at $37^{\circ} \mathrm{C}$ for 30 minutes. The samples were washed and resuspended in ALDEFLUOR buffer. Parallel aliquots of DEAB-treated ALDEFLUOR-stained cell suspensions from each sample were included to confirm ALDH activity.

Histopathology. Whole eyes were obtained from freshly euthanized mice, stored in $10 \%$ formalin, processed by the standard methods, and embedded in paraffin. Sections $(5-\mu \mathrm{m})$ were processed and stained with Martius Scarlet Blue Stain (MSB) and H\&E (Pathology Core Laboratories). Images of the bulbar conjunctiva were taken using the Olympus Inverted Microscope CKX41 and Infinity1-2CB/2MP Digital Color Camera (Olympus America Inc).

Immunofluorescence microscopy. Conjunctival tissues excised from mice were fixed in $4 \%(\mathrm{v} / \mathrm{v})$ paraformaldehyde (PFA, Sigma-Aldrich) for 1.5 hours and thoroughly washed. The samples were then blocked 
with 5\% goat serum (Sigma-Aldrich) for 30 minutes and subsequently stained with rabbit anti-mouse ALDH1 (Abcam) and rat anti-mouse MHC-II (eBioscience) primary antibody in 5\% (v/v) goat serum (Invitrogen) for 48 hours at $4^{\circ} \mathrm{C}$ in the dark. Samples were thoroughly washed and stained with goat anti-rabbit Alexa fluor 633 (Invitrogen) and goat anti-rat Alexa fluor 488 (Invitrogen) secondary antibody in $5 \%(\mathrm{v} / \mathrm{v})$ goat serum and incubated for 1 hour at room temperature. Samples were thoroughly washed and mounted with DAPI-containing media (Vector Laboratories). Images were captured using a Leica SP5 (Leica Microsystems), followed by image analysis with Leica LAS AF lite software.

Statistics. Experiments conducted in vivo were carried out on groups of mice containing $n=5$ mice, or as specified in the figure legends. The experiments that were carried out in vitro were conducted on cultures explanted from at least 3 separate mice. Overall, experiments were carried out on at least 2 separate occasions. Box and whisker plots use the standard method (Prism, GraphPad). Specifically, whiskers indicate the min and max, the hinges indicate the 25th to 75th percentiles, and the middle line is plotted at the median. For statistical analysis, the 1-way ANOVA was used with Bonferroni correction to obtain SEM and statistical significance. A $P$ value of less than 0.05 was considered significant.

Study approval. Duke Institutional Animal Care and Use Committee approved all animal procedures. All animals were treated according to the ARVO Statement for the Use of Animals in Ophthalmic and Vision Research, NIH guidelines on the care and use of animals, and the American Veterinary Medical Association (AVMA) Panel on Euthanasia.

\section{Author Contributions}

SDA executed experimental design and carried out all experiments, data analyses, data interpretation, and manuscript writing. RM and NJR carried out experiments and were involved in data analysis. PSM and SWC developed fibrosis reagents and formulations described and were involved in clinical analysis. VLC procured funding and assisted in experimental design and manuscript writing. DRS was the principal investigator, procured funding, and assisted with experimental design, analysis, interpretation, and manuscript writing.

\section{Acknowledgments}

The authors would like to acknowledge John KG Dart (Moorfields Eye Hospital, UCL Institute of Ophthalmology) for helpful discussions on various topics relevant to this manuscript, including the pathobiology of conjunctival scarring. This study was funded by an R01 from the National Eye Institute/NIH (DRS); Research to Prevent Blindness (DRS); P31 from the National Eye Institute/NIH (Core Grant); and UCL Business Proof-of-Concept (VLC).

Address correspondence to: Daniel R. Saban, Assistant Professor of Ophthalmology and Immunology, 2351 Erwin Road, Durham, North Carolina 27705, USA. Phone: 919.660.0404; E-mail: daniel.saban@duke.edu.

1. Lee CG, et al. Interleukin-13 induces tissue fibrosis by selectively stimulating and activating transforming growth factor beta(1). J Exp Med. 2001;194(6):809-821.

2. Wynn TA. Common and unique mechanisms regulate fibrosis in various fibroproliferative diseases. J Clin Invest. 2007;117(3):524-529.

3. Chiaramonte MG, Donaldson DD, Cheever AW, Wynn TA. An IL-13 inhibitor blocks the development of hepatic fibrosis during a T-helper type 2-dominated inflammatory response. J Clin Invest. 1999;104(6):777-785.

4. Leonardi A, Cortivo R, Fregona I, Plebani M, Secchi AG, Abatangelo G. Effects of Th2 cytokines on expression of collagen, MMP-1, and TIMP-1 in conjunctival fibroblasts. Invest Ophthalmol Vis Sci. 2003;44(1):183-189.

5. Saw VP, et al. Conjunctival interleukin-13 expression in mucous membrane pemphigoid and functional effects of interleukin-13 on conjunctival fibroblasts in vitro. Am J Pathol. 2009;175(6):2406-2415.

6. Saw VP, et al. Profibrotic phenotype of conjunctival fibroblasts from mucous membrane pemphigoid. Am J Pathol. 2011;178(1):187-197.

7. Okuno M, et al. Retinoids exacerbate rat liver fibrosis by inducing the activation of latent TGF-beta in liver stellate cells. Hepatology. 1997;26(4):913-921.

8. Okuno M, et al. Retinoids in liver fibrosis and cancer. Front Biosci. 2002;7:d204-d218.

9. Rankin AC, Hendry BM, Corcoran JP, Xu Q. An in vitro model for the pro-fibrotic effects of retinoids: mechanisms of action. Br J Pharmacol. 2013;170(6):1177-1189.

10. Hellemans K, et al. Differential modulation of rat hepatic stellate phenotype by natural and synthetic retinoids. Hepatology. 2004;39(1):97-108.

11. Varani J, et al. All-trans retinoic acid stimulates growth and extracellular matrix production in growth-inhibited cultured human skin fibroblasts. J Invest Dermatol. 1990;94(5):717-723. 
12. Davis BH, Kramer RT, Davidson NO. Retinoic acid modulates rat Ito cell proliferation, collagen, and transforming growth factor beta production. J Clin Invest. 1990;86(6):2062-2070.

13. Seifert WF, et al. Vitamin A deficiency potentiates carbon tetrachloride-induced liver fibrosis in rats. Hepatology. 1994;19(1):193201.

14. He H, Mennone A, Boyer JL, Cai SY. Combination of retinoic acid and ursodeoxycholic acid attenuates liver injury in bile duct-ligated rats and human hepatic cells. Hepatology. 2011;53(2):548-557.

15. Iwata M, Hirakiyama A, Eshima Y, Kagechika H, Kato C, Song SY. Retinoic acid imprints gut-homing specificity on T cells. Immunity. 2004;21(4):527-538.

16. Agace WW, Persson EK. How vitamin A metabolizing dendritic cells are generated in the gut mucosa. Trends Immunol. 2012;33(1):42-48.

17. Bakdash G, Vogelpoel LT, van Capel TM, Kapsenberg ML, de Jong EC. Retinoic acid primes human dendritic cells to induce gut-homing, IL-10-producing regulatory T cells. Mucosal Immunol. 2015;8(2):265-278.

18. Coombes JL, et al. A functionally specialized population of mucosal CD103+ DCs induces Foxp3+ regulatory $\mathrm{T}$ cells via a TGF-beta and retinoic acid-dependent mechanism. J Exp Med. 2007;204(8):1757-1764.

19. Hall JA, Grainger JR, Spencer SP, Belkaid Y. The role of retinoic acid in tolerance and immunity. Immunity. 2011;35(1):13-22.

20. Sun CM, et al. Small intestine lamina propria dendritic cells promote de novo generation of Foxp3 T reg cells via retinoic acid J Exp Med. 2007;204(8):1775-1785.

21. Klebanoff CA, et al. Retinoic acid controls the homeostasis of pre-cDC-derived splenic and intestinal dendritic cells. J Exp Med. 2013;210(10):1961-1976.

22. Levin AA, et al. 9-cis retinoic acid stereoisomer binds and activates the nuclear receptor RXR alpha. Nature. 1992;355(6358):359-361.

23. Chambon P. A decade of molecular biology of retinoic acid receptors. FASEB J. 1996;10(9):940-954.

24. Guilliams M, et al. Skin-draining lymph nodes contain dermis-derived CD103(-) dendritic cells that constitutively produce retinoic acid and induce Foxp3(+) regulatory T cells. Blood. 2010;115(10):1958-1968.

25. Yokota A, et al. GM-CSF and IL-4 synergistically trigger dendritic cells to acquire retinoic acid-producing capacity. Int Immunol. 2009;21(4):361-377.

26. Heng TS, Painter MW, Immunological Genome Project Consortium. The Immunological Genome Project: networks of gene expression in immune cells. Nat Immunol. 2008;9(10):1091-1094.

27. Foster CS, Calonge M. Atopic keratoconjunctivitis. Ophthalmology. 1990;97(8):992-1000.

28. Guilliams M, et al. Dendritic cells, monocytes and macrophages: a unified nomenclature based on ontogeny. Nat Rev Immunol. 2014;14(8):571-578.

29. Ahadome SD, et al. Aldehyde dehydrogenase inhibition blocks mucosal fibrosis in human and mouse ocular scarring. JCI Insight. 2016;1(12):e87001

30. Khandelwal P, et al. Ocular mucosal CD11b+ and CD103+ mouse dendritic cells under normal conditions and in allergic immune responses. PLOS ONE. 2013;8(5):e64193.

31. Mayer CT, et al. Selective and efficient generation of functional Batf3-dependent CD103+ dendritic cells from mouse bone marrow. Blood. 2014;124(20):3081-3091.

32. Helft J, et al. GM-CSF Mouse Bone Marrow Cultures Comprise a Heterogeneous Population of CD11c(+)MHCII(+) Macrophages and Dendritic Cells. Immunity. 2015;42(6):1197-1211.

33. Schlereth S, Lee HS, Khandelwal P, Saban DR. Blocking CCR7 at the ocular surface impairs the pathogenic contribution of dendritic cells in allergic conjunctivitis. Am J Pathol. 2012;180(6):2351-2360.

34. Saban DR. The chemokine receptor CCR7 expressed by dendritic cells: a key player in corneal and ocular surface inflammation. Ocul Surf. 2014;12(2):87-99.

35. Minshall EM, et al. Eosinophil-associated TGF-beta1 mRNA expression and airways fibrosis in bronchial asthma. Am J Respir Cell Mol Biol. 1997;17(3):326-333.

36. Connolly MK, et al. In liver fibrosis, dendritic cells govern hepatic inflammation in mice via TNF-alpha. J Clin Invest. 2009;119(11):3213-3225.

37. Kitamura $\mathrm{H}$, et al. Mouse and human lung fibroblasts regulate dendritic cell trafficking, airway inflammation, and fibrosis through integrin $\alpha v \beta 8$-mediated activation of TGF- $\beta$. J Clin Invest. 2011;121(7):2863-2875.

38. Schlitzer A, et al. Identification of cDC1- and cDC2-committed DC progenitors reveals early lineage priming at the common DC progenitor stage in the bone marrow. Nat Immunol. 2015;16(7):718-728.

39. Plantinga $\mathrm{M}$, et al. Conventional and monocyte-derived $\mathrm{CD} 11 \mathrm{~b}(+)$ dendritic cells initiate and maintain $\mathrm{T}$ helper 2 cell-mediated immunity to house dust mite allergen. Immunity. 2013;38(2):322-335.

40. Gao N, Yin J, Yoon GS, Mi QS, Yu FS. Dendritic cell-epithelium interplay is a determinant factor for corneal epithelial wound repair. Am J Pathol. 2011;179(5):2243-2253.

41. Jiao J, et al. Dendritic cell regulation of carbon tetrachloride-induced murine liver fibrosis regression. Hepatology. 2012;55(1):244-255.

42. Chiba T, et al. Retinoic Acid Signaling Coordinates Macrophage-Dependent Injury and Repair after AKI. J Am Soc Nephrol. 2016;27(2):495-508.

43. Dutertre CA, Wang LF, Ginhoux F. Aligning bona fide dendritic cell populations across species. Cell Immunol. 2014;291(1-2):3-10. 